\title{
Beam Expansion of Blind Spot Detection Radar Antennas Using a Radome with Defected Corrugated Inner Wall
}

\author{
Hayeon Kim, ${ }^{1}$ Haengseon Lee, ${ }^{1}$ Jeonghoon Cho, ${ }^{2}$ and Cheolbok Kim ${ }^{2}$ \\ ${ }^{1}$ Department of Electronic Engineering, Sogang University, 1 Sinsu-dong, Mapo-gu, Seoul, Republic of Korea \\ ${ }^{2}$ Applied Network Team, LG Innotek Co., Ltd., RङD Center 1271, Sa-3-dong, Sangrok-gu, Ansan-si, Gyeonggi-do, Republic of Korea
}

Correspondence should be addressed to Haengseon Lee; leehs95@sogang.ac.kr

Received 31 May 2017; Accepted 11 September 2017; Published 10 December 2017

Academic Editor: N. Nasimuddin

Copyright (C) 2017 Hayeon Kim et al. This is an open access article distributed under the Creative Commons Attribution License, which permits unrestricted use, distribution, and reproduction in any medium, provided the original work is properly cited.

\begin{abstract}
A beam expanding radome for $76.5 \mathrm{GHz}$ automotive radar antennas is presented whose inner surface is engraved with corrugations. The radar used for blind spot detection (BSD) requires a very wide beam width to ensure longer time for tracking out-of-sight objects. It is found that the corrugations modulate the phase velocities of the waves along the surface, which increases beam width in the far field. In addition, defects in the corrugation increase beam width even further. The presented structure satisfies the beam width requirement while keeping a low profile.
\end{abstract}

\section{Introduction}

As safety becomes an important issue in car industries and autonomous vehicle technologies, automotive radars are given much interest. To assist drivers, various automotive radars are being developed such as those for long range, middle range, short range, and blind spot detection. The design requirements for those radars become different following the application of them. While the long-range radars require high gain and narrow beam widths, those for blind spot detection require very wide beam widths. Radar components like antennas and radomes are designed to satisfy those specifications. Among those, radomes have been used to protect antennas from corrosion due to rain, wind, extreme temperature, and so on. At the same time, their influence on the radiation patterns of enclosed antennas should be minimized [1].

Commonly used radome types are of style $a$ and style $b$ [2]. The former has thicknesses of integral multiple of half wavelength, and the latter has thin walls less than 0.1 wavelength. Other styles have multilayer structures to decrease reflections for a wide range of incidence angles and bandwidths.

To reduce the reflected waves, similar researches have been carried out on the junction of waveguide sections containing different dielectric materials. For the junction of hollow waveguide and that containing dielectric of refractive index $n$, a quarter wavelength transformer containing dielectric with refractive index of $\sqrt{n}$ is frequently used to decrease mismatches [3]. Instead of using a different medium, corrugations, arrays of small cylinders, and holes are formed on the surface of dielectric which act as if they were a medium with lower refractive indices [4].

Similar approaches have been tried for radomes. For a radome material of refractive index $n$, a quarter wavelength thickness dielectric layer is coated whose refractive index is $\sqrt{n}$ to decrease mismatches [5]. In [6-8], inhomogeneous radome walls are used which have continuously varying refractive index profile. Instead of attaching a different dielectric layer, corrugations are formed on the surface of radomes $[9,10]$. By changing the ratio of dielectric and air portions, effective refractive index can be set to the value $\sqrt{n}$ which minimizes reflections.

In this paper, a beam-width-expanding radome design is presented. For simple fabrication, a single layer radome with corrugated inner wall is investigated. Relationship between field distributions on the radome surface and far field radiation patterns is found. Parametric studies show that the magnitude and phase variations at the surface of the radome wall determine beam widths of the radiation pattern. 


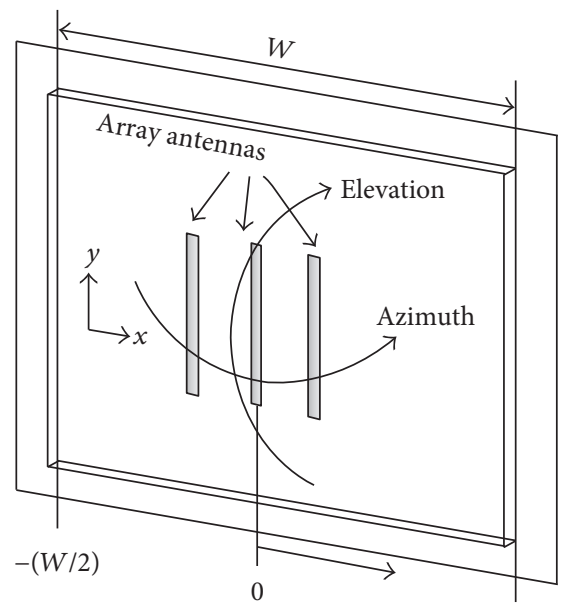

$(W / 2)$

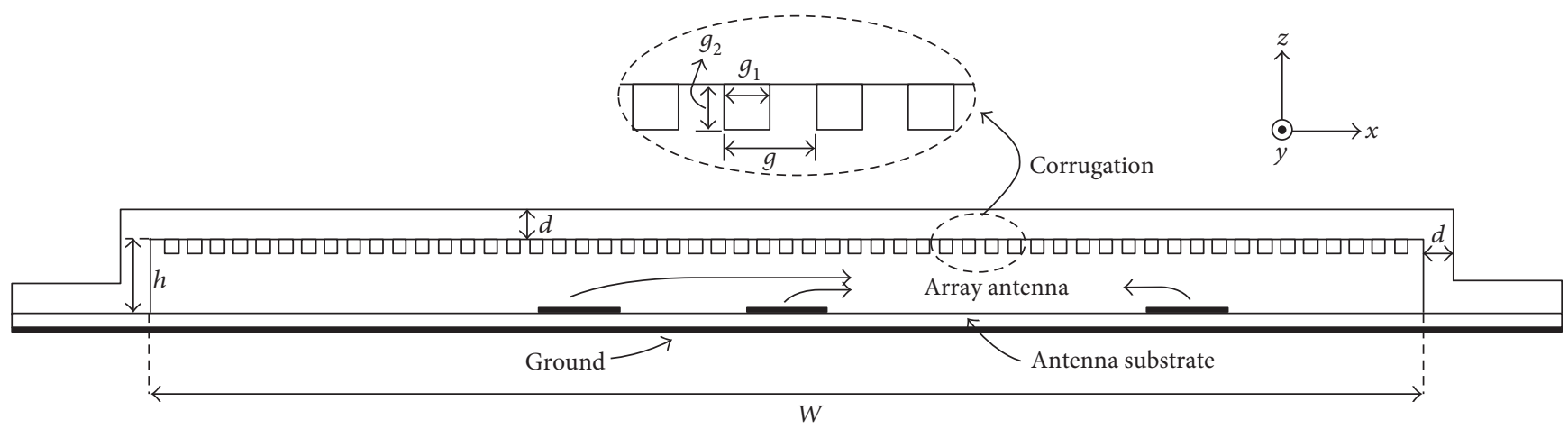

FIgURE 1: Corrugated radome.

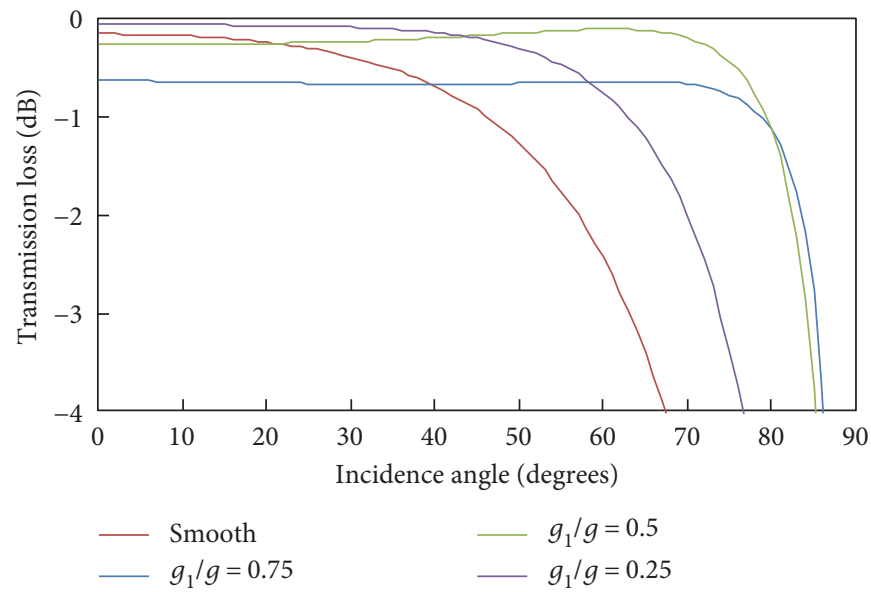

Figure 2: Comparison of transmission loss at $76.5 \mathrm{GHz}$ for different $g_{1} / g$ ratios.

It is found that fast phase variation near the enclosed antenna increases the beam width. In addition, slow variation of magnitude near the antenna increases beam width even further. In the following sections, experimental results and full-wave simulations are given which verify the argument.

\section{Corrugated Radome}

In this section, radome design guidelines are given for blind spot detection radars. Unlike the usual definition of half power beam width, the definition for BSD radars means that 


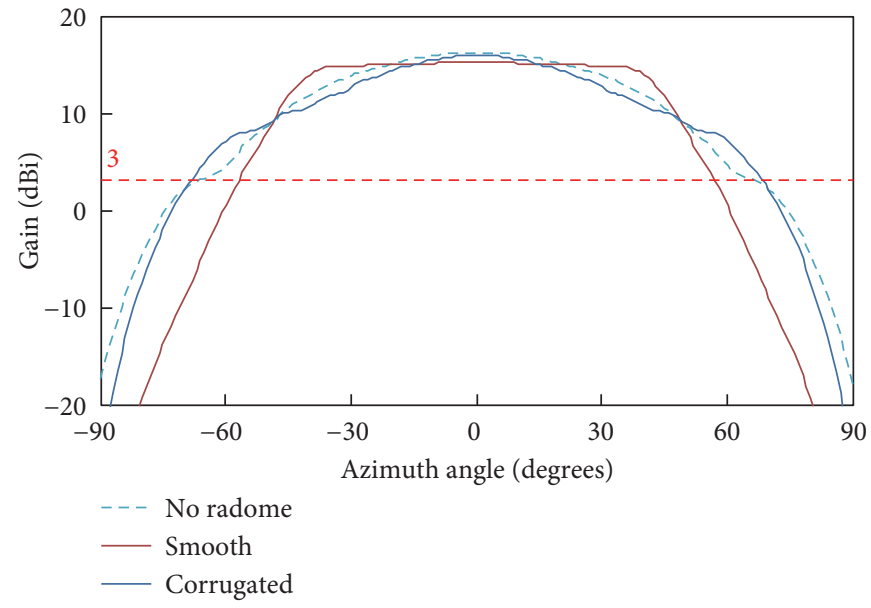

FIGURE 3: $3 \mathrm{dBi}$ beam width comparison: smooth-walled radome versus corrugated radome.

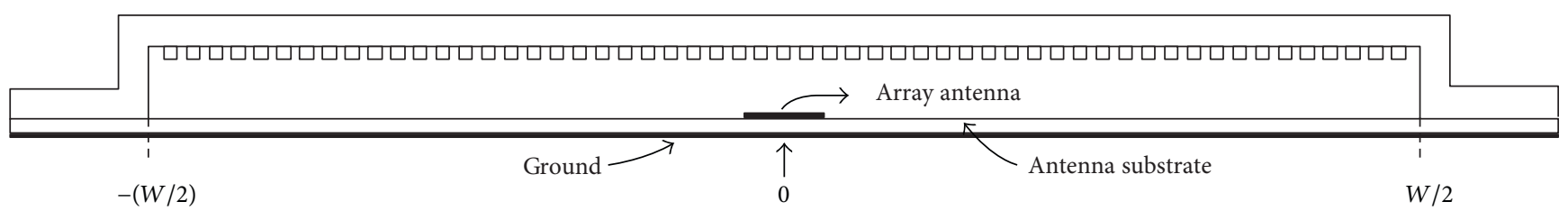

(a) Corrugated radome

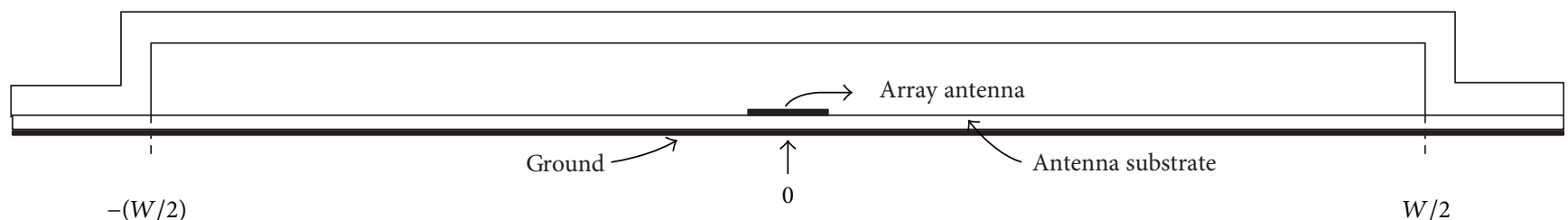

(b) Smooth-walled radome

FIGURE 4: Simulation model for extracting near-field region e-field distribution.

the angular range of the antenna gain is larger than $3 \mathrm{dBi}$. The antennas used for this study are linear patch arrays which have sharp fan beam radiation patterns widely used for automotive radars.

The wider the beam width, the longer the tracking time for invisible objects in the blind spot region. To increase the angular range, discontinuous refractive index profile along the vertical direction should be smoothed, which can be accomplished by attaching additional matching layers with intermediate refractive indices. To alleviate the burden of the antireflection coating, corrugated surfaces can be formed as shown in Figure 1. The effective permittivity of the corrugated surface can be estimated using the following formula [11].

$$
\varepsilon_{\text {cor }}=\varepsilon_{0}+\left(\varepsilon_{\text {radome }}-\varepsilon_{0}\right) \frac{g_{1}}{g}
$$

Approximating the corrugated radome by a stack of multiple layers, transmission loss can be calculated using the formula of [12]. Figure 2 shows the transmission loss at
TABLE 1: $3 \mathrm{dBi}$ beam widths of the different radome surfaces.

\begin{tabular}{lc}
\hline Model & Beam width (degrees) \\
\hline Smooth-walled radome & 114.2 \\
Corrugated radome & 137 \\
\hline
\end{tabular}

$76.5 \mathrm{GHz}$ as a function of $g_{1} / g$ when the thickness of the radome is $1 \mathrm{~mm}$ (half wavelength), and $g_{1}$ ranges from $0.25 \mathrm{~mm}$ to $0.75 \mathrm{~mm}$ with the value of $g=1 \mathrm{~mm}$. To increase the angular range, the value of $g_{1} / g$ should be increased. Although the loss becomes smaller when the ratio is small, the angular range becomes larger when the ratio is large.

The radome substrate is $\mathrm{PBT}+30 \%$ glass (polybutylene terephthalate with $30 \%$ glass fiber) whose relative permittivity is 3.1 at the frequency of $76.5 \mathrm{GHz}$. The wavelength at this frequency in air is $3.92 \mathrm{~mm}$ and that in the radome medium is $2.23 \mathrm{~mm}$. For this simulation, thickness of the radome is set to be $1 \mathrm{~mm}$. The height of the inner wall from the antenna is set to be $2 \mathrm{~mm}$. The width $\left(g_{1}\right)$ and the period $(\mathrm{g})$ of the corrugations are set to $0.5 \mathrm{~mm}$ and $1 \mathrm{~mm}$, which corresponds to the effective permittivity of 2.05. Figure 3 


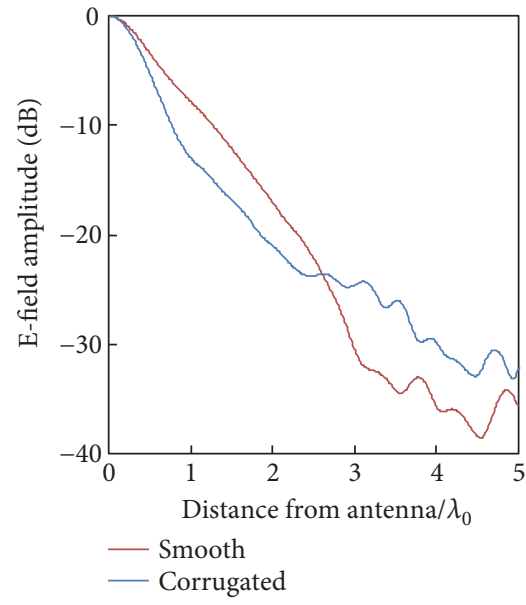

(a) Amplitude

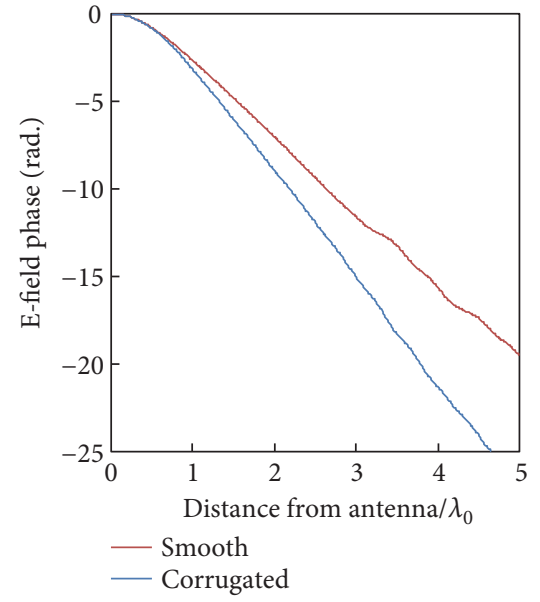

(b) Unwrapped phase

FIgURE 5: Normalized e-field intensity distribution for flat and corrugated radomes: (a) amplitude and (b) unwrapped phase.

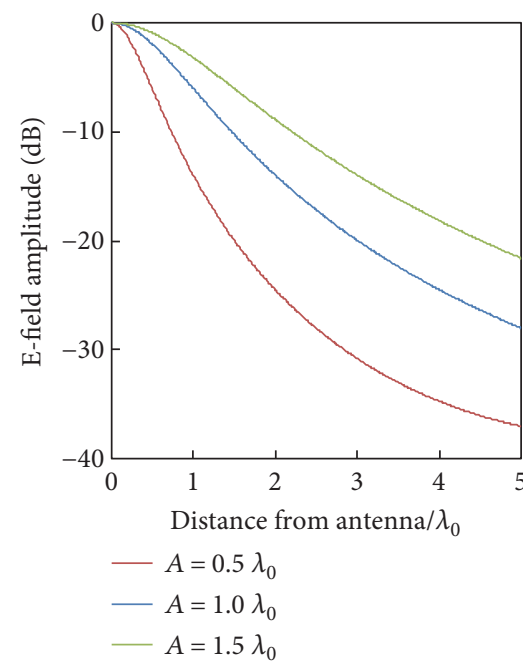

(a) Amplitude

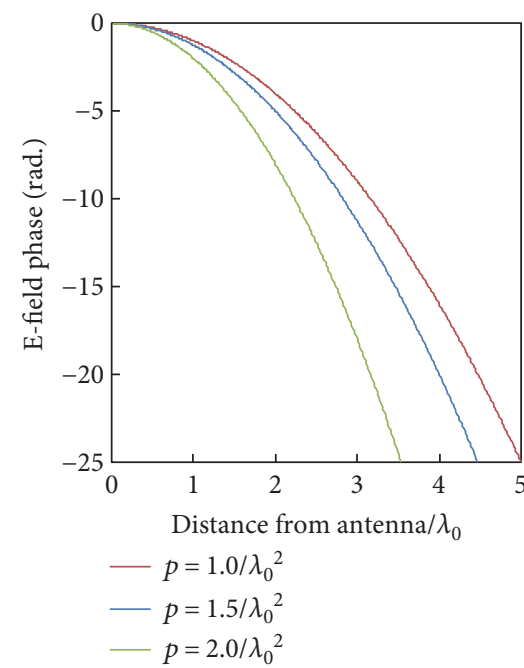

(b) Unwrapped phase

FIGURE 6: Approximated normalized electric field intensity distribution.

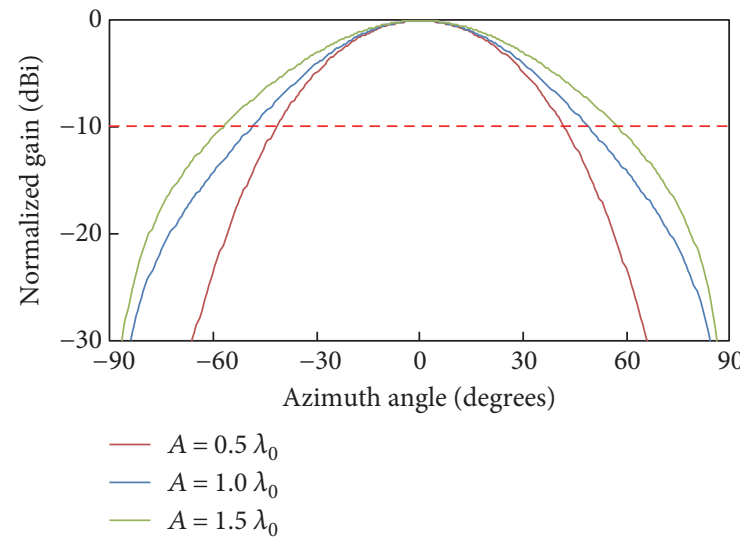

(a) Far-field pattern when $p=2.0 / \lambda_{0}^{2}$ and amplitude function $\Psi(x)$ is varied

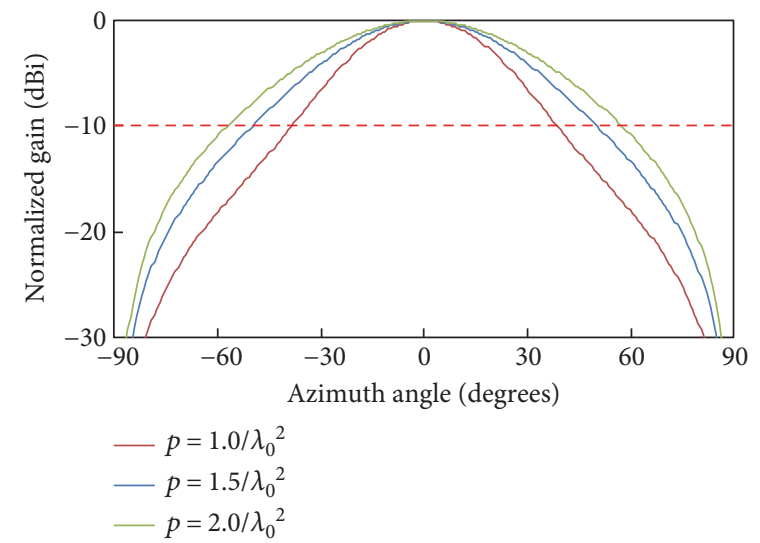

(b) Far-field pattern when $n=2, A=1.5 \lambda_{0}$, and phase function $\phi(x)$ is varied

Figure 7: Normalized far-field patterns calculated using approximated amplitude and phase shown in Figure 6. 


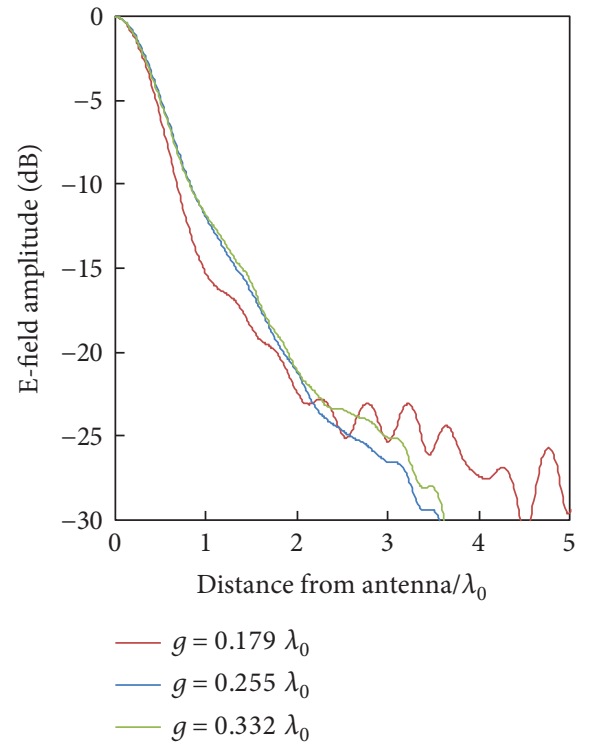

(a) Amplitude

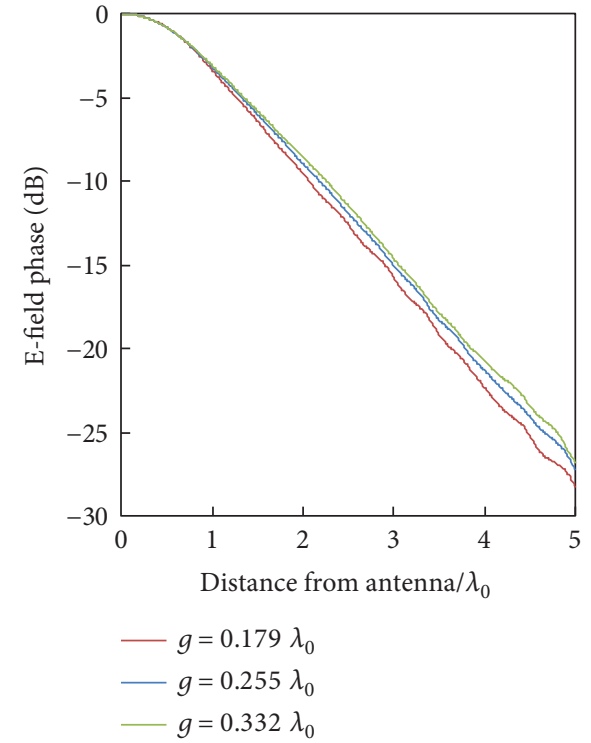

(b) Unwrapped phase

FIGURE 8: Normalized e-field intensity distribution for the different corrugation periods.

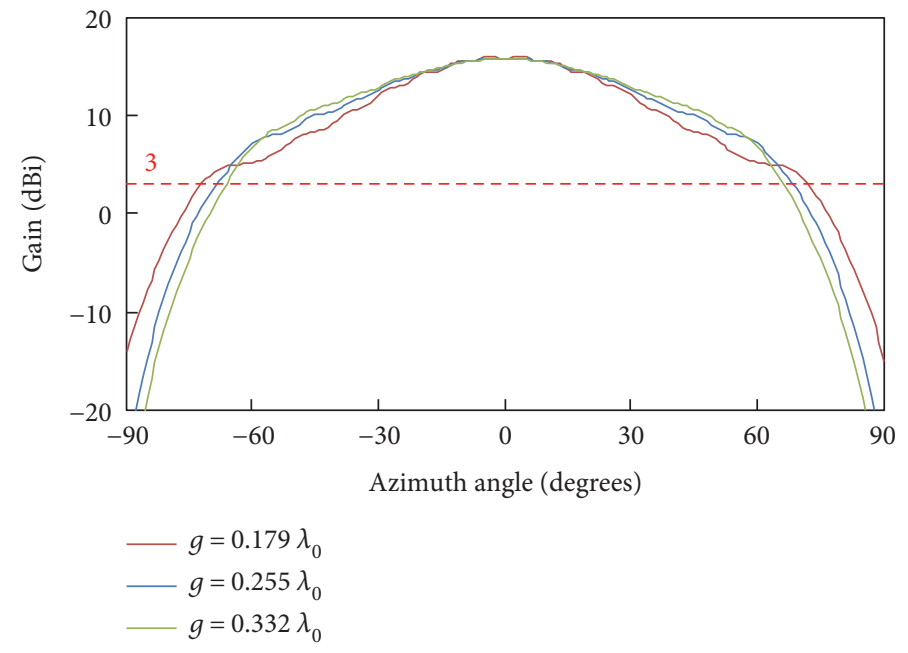

FIGURE 9: $3 \mathrm{dBi}$ beam width comparison for the different corrugation periods.

shows the full-wave simulated radiation patterns of the radomes with the corrugated inner wall and the simple planar wall as shown in Figure 4. The beam width of the radome with a corrugated inner wall is larger than that of the planar inner wall by 20 degrees. Precise beam widths are listed in Table 1.

2.1. Relation between Near-Field Pattern and $3 \mathrm{dBi}$ Beam Width. To find the cause of the increased beam width of the corrugated radome, influences of the near-field distributions on the radiation patterns are studied in terms of magnitudes and phases. It is known that the far-field radiation patterns can be obtained by Fourier transforms of the nearfield distributions as shown by (2) [13]. It is assumed that
TABLE 2: $3 \mathrm{dBi}$ beam widths of the different corrugation periods.

\begin{tabular}{lc}
\hline Model & Beam width (degrees) \\
\hline$g=0.179 \lambda_{0}$ & 144.2 \\
$g=0.255 \lambda_{0}$ & 137 \\
$g=0.332 \lambda_{0}$ & 132.6 \\
\hline
\end{tabular}

the patch array antennas are placed along the $y$-axis and the periodic corrugations are formed along the $x$-axis.

$$
\begin{aligned}
\text { Far field } & =\int_{-(W / 2)}^{W / 2} E(x) e^{j k x \cos \theta} d x, \\
E(x) & =\Psi(x) e^{j \varphi(x)},
\end{aligned}
$$




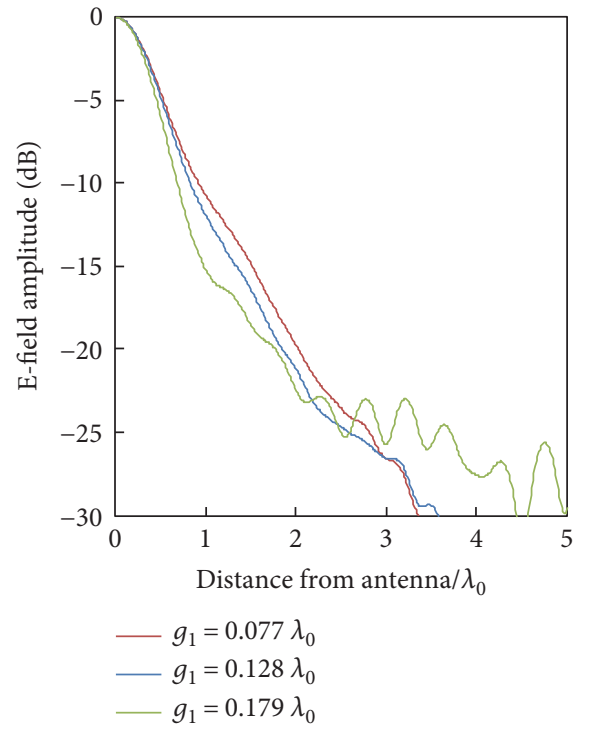

(a) Amplitude

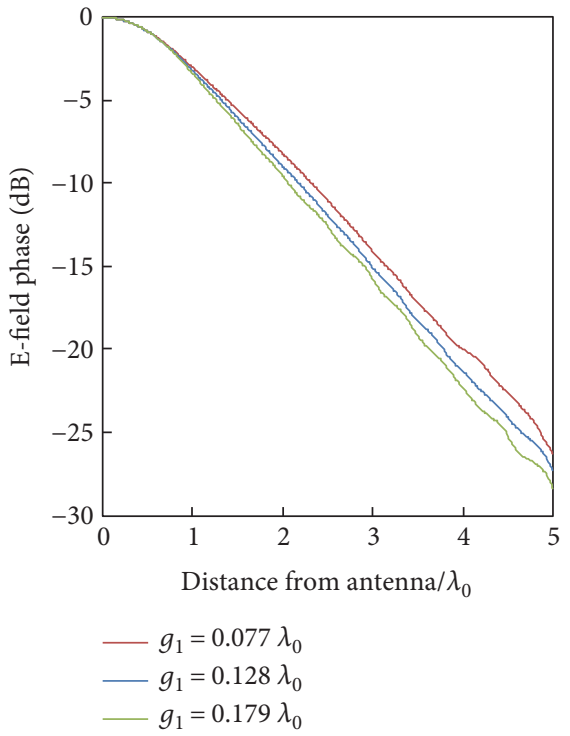

(b) Unwrapped phase

FIGURE 10: Normalized e-field intensity distribution for the different ridge widths.

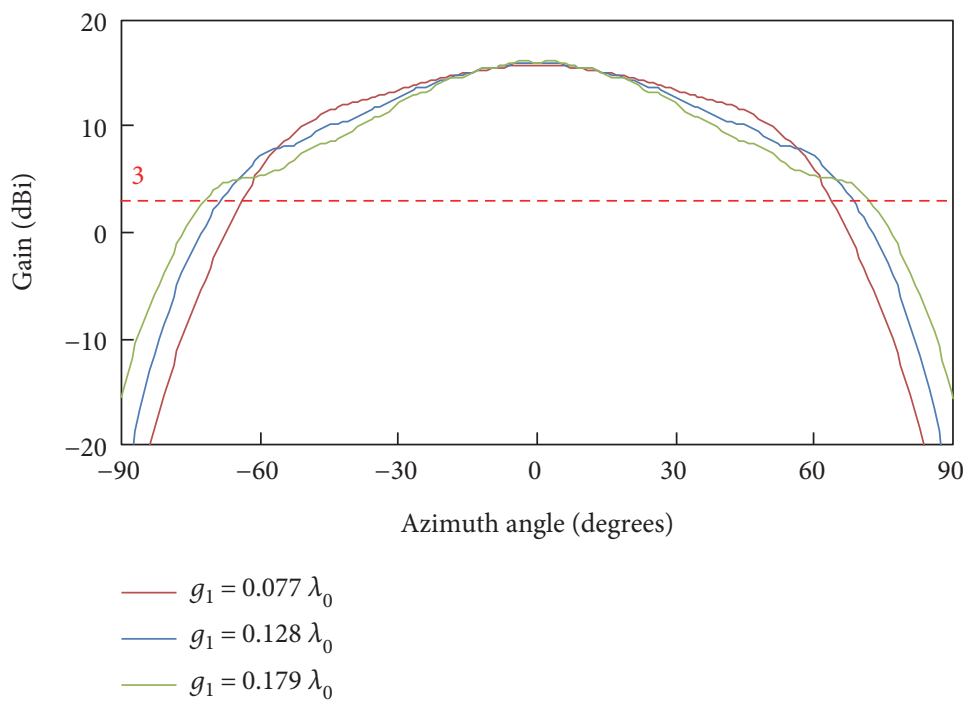

FIGURE 11: $3 \mathrm{dBi}$ beam width comparison for the different ridge widths.

where $E(x)$ is the near-field electric field, $\Psi(x)$ is the magnitude, and $\phi(x)$ is the phase function. The integration is performed over the range from $-(W / 2)$ to $W / 2$. The antenna is assumed to be at the origin.

Figure 5 shows the near-field distributions of the electric fields of the two radomes of Figure 4 . The fields are sampled at every $0.05 \mathrm{~mm}$ along the $x$-axis from 0 , which is marked in Figure 1 , and $0.1 \mathrm{~mm}$ above the radome surfaces. Due to the symmetry about the antenna, only the fields along the positive $x$-axis are recorded. It is found that the phase function of the corrugated radome which has a large beam width has a steep slope. The magnitude of the near-field distribution of the corrugated radome also has a steep slope than that of the smooth one.
TABLE 3: $3 \mathrm{dBi}$ beam widths of the different ridge widths.

\begin{tabular}{lc}
\hline Model & Beam width (degrees) \\
\hline$g_{1}=0.077 \lambda_{0}$ & 128 \\
$g_{1}=0.128 \lambda_{0}$ & 137 \\
$g_{1}=0.179 \lambda_{0}$ & 144.2 \\
\hline
\end{tabular}

To investigate the impact of the near-field phase and magnitude on the radiation pattern, parametric equations for those two quantities are devised as follows:

$$
\Psi(x)=\frac{1}{\left\{1+(|x| / A)^{n}\right\}^{2}},
$$




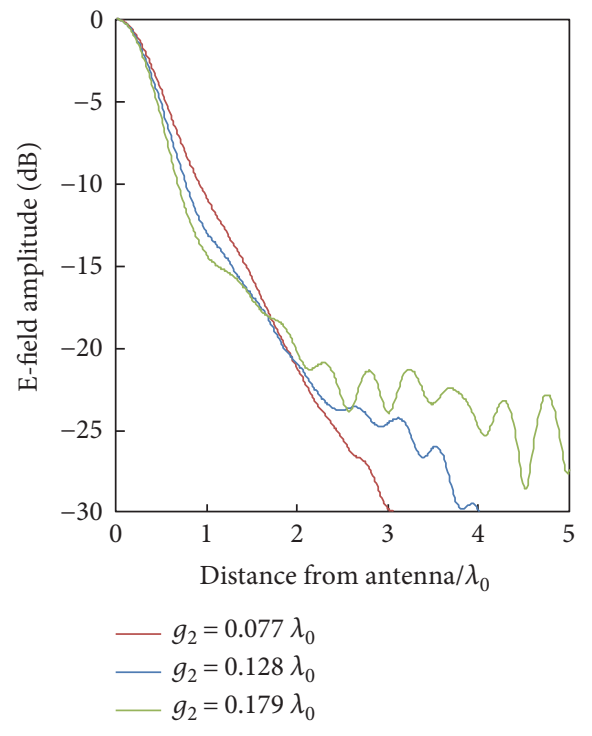

(a) Amplitude

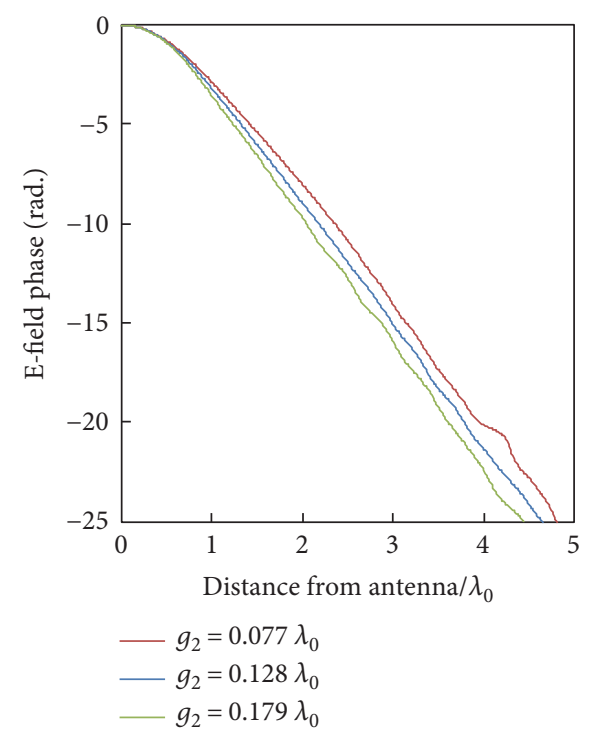

(b) Unwrapped phase

FIGURE 12: Normalized e-field intensity distribution for the different corrugation depths.

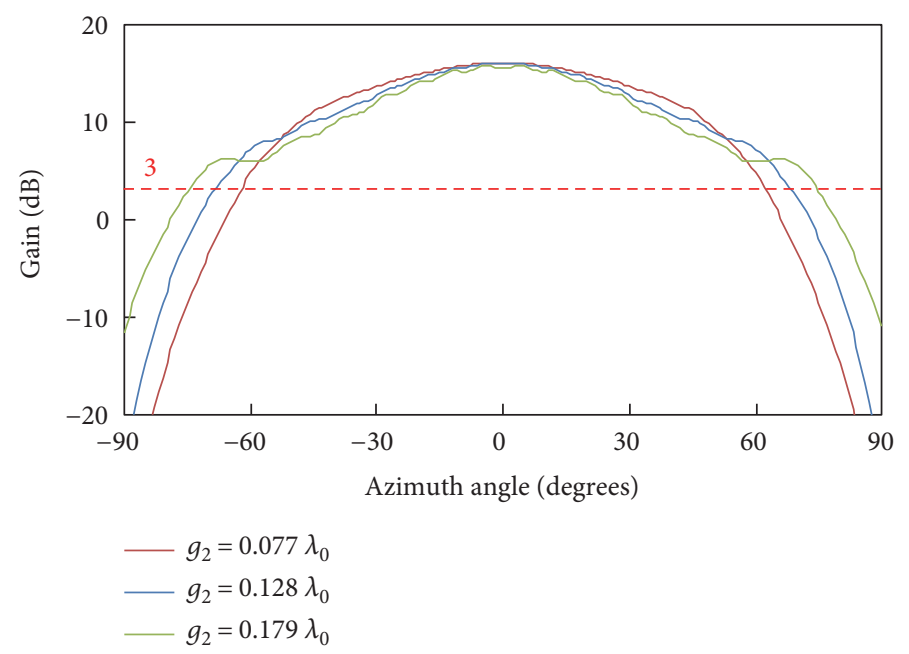

FIGURE 13: The $3 \mathrm{dBi}$ beam width comparison for the different corrugation depths.

$$
\phi(x)=-p x^{2}
$$

The constant $A$ determines the extent beyond which the magnitude begin to decrease. For phase function (5), $p$ is a parameter whose unit is radian per wavelength squared. With large $p$, phase function decreases more rapidly.

Figure 6(a) shows the magnitude distributions of the electric field on the radome surface with $n$ fixed and $A$ changed. Figure 6(b) shows the phase distributions with parameter $p$ changed. Figure 7 shows the far-field radiation patterns along the azimuth direction for various sets of amplitude and phase parameters. Figure 7(a) shows the radiation patterns with parameter $A$ changed. The value of $n$ is fixed to be 2 , and the phase parameter $p$ is fixed to be $2.0 / \lambda_{0}{ }^{2}$. Figure $7(\mathrm{~b})$
TABLE 4: $3 \mathrm{dBi}$ beam widths of the different corrugation depths.

\begin{tabular}{lc}
\hline Model & Beam width (degrees) \\
\hline$g_{2}=0.077 \lambda_{0}$ & 124.8 \\
$g_{2}=0.128 \lambda_{0}$ & 137 \\
$g_{2}=0.179 \lambda_{0}$ & 149.6 \\
\hline
\end{tabular}

shows the radiation patterns with parameter $p$ changed from $1.0 / \lambda_{0}{ }^{2}$ to $2.0 / \lambda_{0}{ }^{2}$ with fixed amplitude parameters of $n=2$ and $A=1.5 \lambda_{0}$. It is found that the near-field distribution with slowly decreasing magnitude and rapidly varying phase has wide beam widths. 


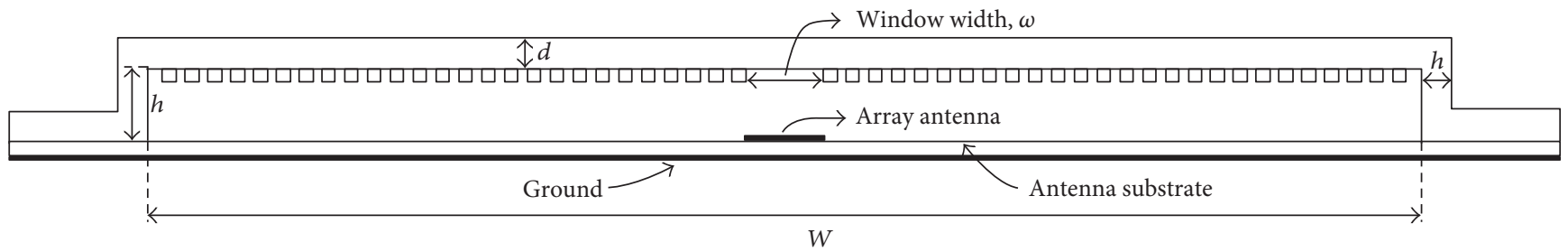

Figure 14: Defected corrugated radome (side view).

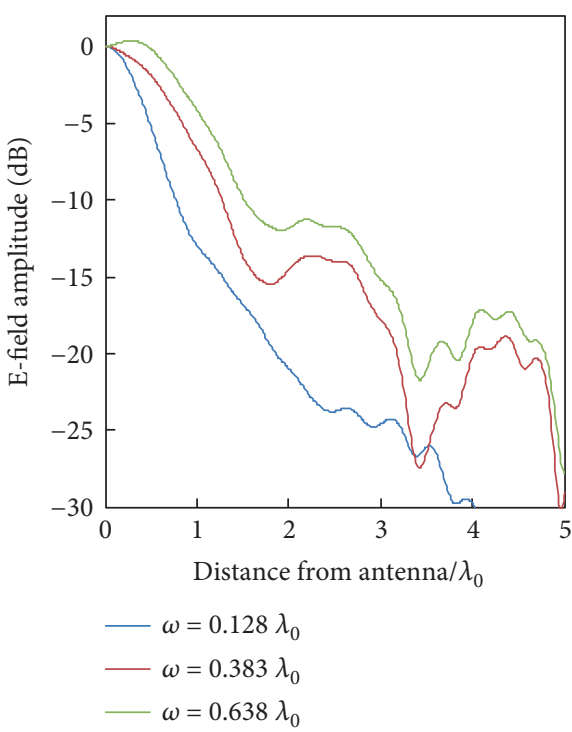

(a) Amplitude

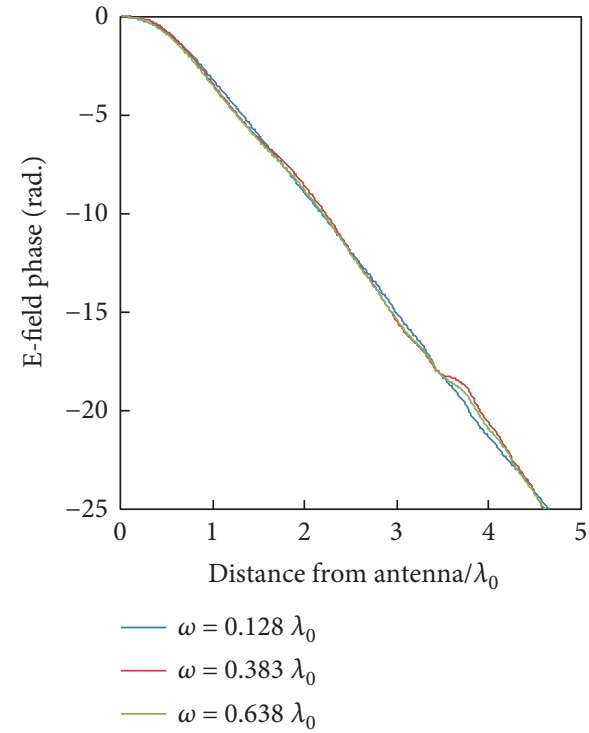

(b) Unwrapped phase

FIgURE 15: Normalized e-field intensity distribution for defected corrugated radomes.

2.2. Impact of Shape Parameters of Corrugation on Beam Width. In the preceding section, it is shown that near-field distributions with slowly varying magnitude and rapidly varying phase produce large beam width. To implement the desired near-field distribution by corrugated surfaces, influences of the period $(g)$, width of the ridge $\left(g_{1}\right)$, and depth $\left(g_{2}\right)$ of the corrugations are observed using full-wave simulations. While $g, g_{1}$, and $g_{2}$ are varied, the thickness $(d)$ and height $(h)$ of the radome as shown in Figure 1 are fixed to be $1 \mathrm{~mm}$ and $2 \mathrm{~mm}$, respectively.

2.2.1. Impact of Corrugation Period. The period of a corrugation $(g)$ is made up of the ridge $\left(g_{1}\right)$ and trough. As the period $(g)$ is varied, near-field distribution changes as shown in Figure 8. With small period, magnitude and phase change rapidly with increasing distance from the radiating antenna, which yields large beam width as shown in Figure 9. Table 2 summarizes the $3 \mathrm{dBi}$ beam width with changing periods.

2.2.2. Impact of Ridge Width. Ridge width $g_{1}$ is varied while $g$ is fixed. As the width grows, as shown in Figure 10, the magnitude and phase distribution of the electric field change steeply. In addition, it contributes to large beam width as shown in Figure 11. The $3 \mathrm{dBi}$ beam widths with varying ridge width are summarized in Table 3.

2.2.3. Impact of Corrugation Depth. The impact of corrugation depth $\left(g_{2}\right)$ on the near-field distribution is also observed. The ratio of $g_{1} / g$ is fixed to be 0.5 . As the depth increases from $0.077 \lambda_{0}$ to $0.179 \lambda_{0}$, magnitude and phase in the near field vary rapidly as shown in Figure 12. Far-field radiation patterns are shown in Figure 13. As $g_{2}$ approaches $\lambda_{0} / 4$, however, beam width decreases. When the magnitude varies rapidly, ripples of the far zone radiation pattern increase. The $3 \mathrm{dBi}$ beam widths of each cases are listed in Table 4.

\section{Defected Corrugated Surface}

In Section 2.1, the condition for large beam width is derived such that slowly varying magnitude and rapidly varying phase distribution are needed. However, the condition cannot be achieved by varying the shape parameters of the corrugation only. In this section, introduction of defects in the periodic structure is considered as shown in Figure 14. 


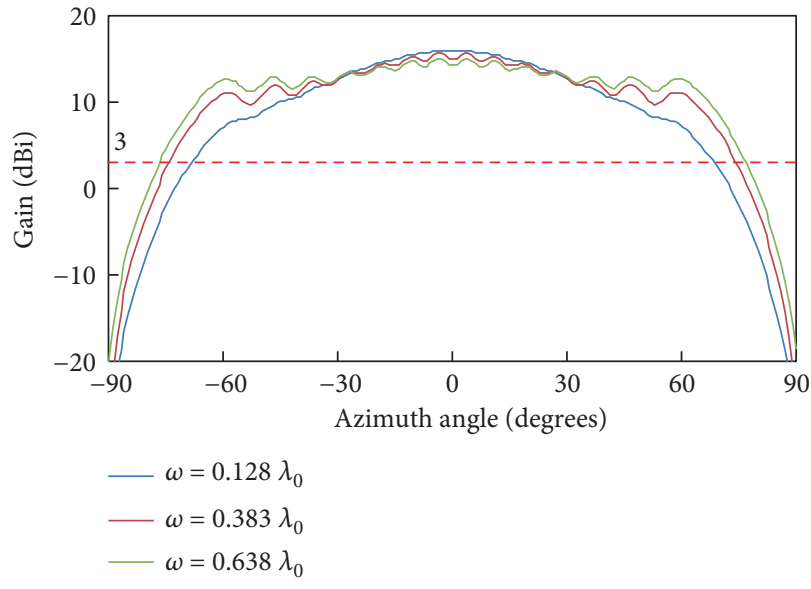

(a) Azimuth pattern

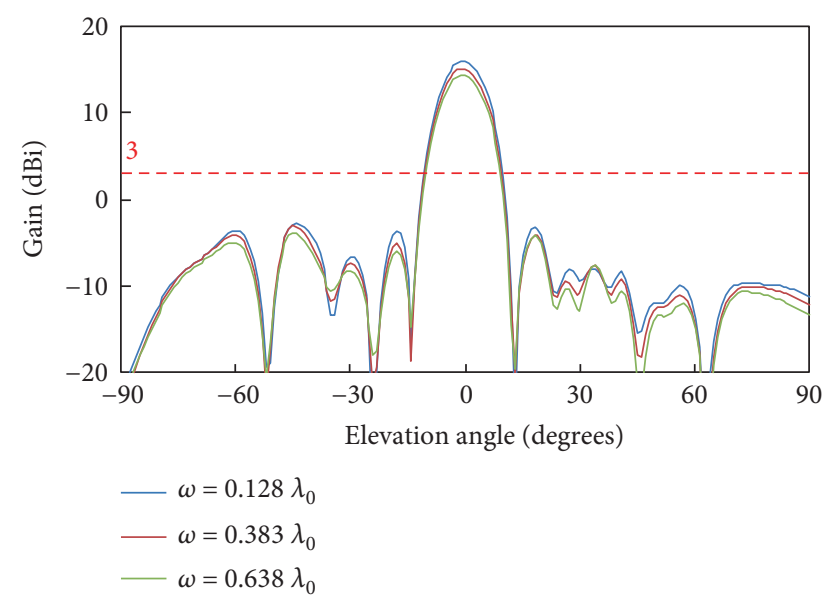

(b) Elevation pattern

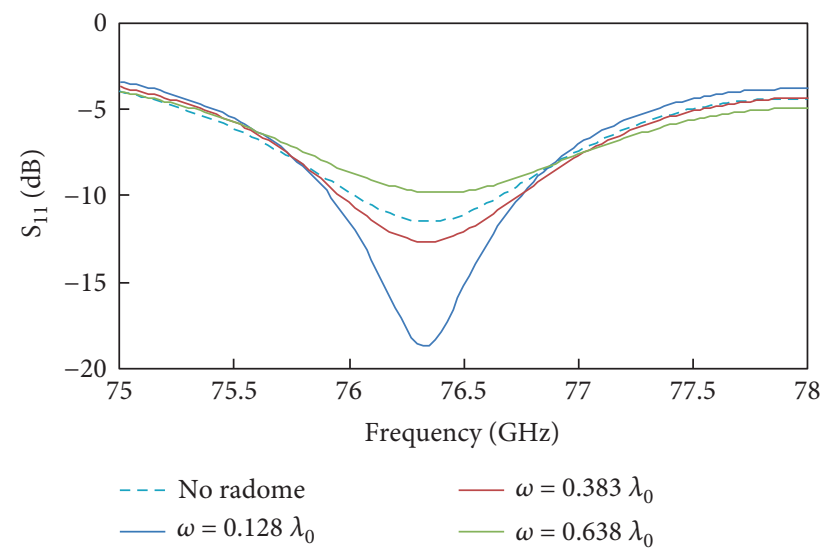

(c) Input reflection loss, $S_{11}$

FIGURE 16: Radiation pattern and input reflection loss comparison for various window widths.

In the preceding section, magnitude variation of a smoothwalled radome is slower than that of a corrugated wall. After removing a corrugation above the antenna, the behavior of the near-field distribution is observed.

Figure 15 shows the near-field distributions with varying width of the defects above the radiating antenna. Those defects are made by removing one to two corrugations above the antenna. Although the phase distribution does not show noticeable change, the magnitude variation becomes slower near the defect.

Figures 16(a) and 16(b) show the full-wave simulated radiation patterns of the defected corrugated surface. As the window size (defected region) becomes comparable to one wavelength, the azimuth beam width reaches maximum. If increased further, beam width decreased. While the beam widths along the azimuthal direction are affected by the window size, those along the elevation angle remain almost unaltered. Table 5 summarizes the $3 \mathrm{dBi}$ beam widths along the azimuthal and elevation directions of the defected corrugated radomes with different $\omega$.

Figure 16(c) shows the input return losses of the antenna with no radome and with radomes with different window widths. When $\omega$ is $0.638 \lambda_{0}, S_{11}$ is $-9.7 \mathrm{~dB}$. Other than this case, input reflection losses remain below $-10 \mathrm{~dB}$.
TABLE 5: $3 \mathrm{dBi}$ beam widths of the defected corrugated radomes with different $\omega$.

\begin{tabular}{lcc}
\hline Model & $\begin{array}{c}\text { Azimuth beam } \\
\text { width (degrees) }\end{array}$ & $\begin{array}{c}\text { Elevation beam } \\
\text { width (degrees) }\end{array}$ \\
\hline$\omega=0.128 \lambda_{0}$ (no defect) & 137 & 20.6 \\
$\omega=0.383 \lambda_{0}$ & 148.5 & 20.1 \\
$\omega=0.638 \lambda_{0}$ & 153.6 & 19.5 \\
\hline
\end{tabular}

\section{Measurement Results}

Using the design guidelines in the preceding sections, radomes and automotive radar antennas are built as shown in Figures 17 and 18. In Figure 17, three types of radomes having different inner surfaces are shown. They have different thicknesses $(d)$ and heights $(h)$, which are summarized in Table 6 along with measured beam width. The radome with defected corrugation has five defects on the corrugated surface to increase beam widths. The widths of those defects are $2.5 \mathrm{~mm}$. The radar antennas are composed of one transmitting antenna and four receiving antennas. The antennas 


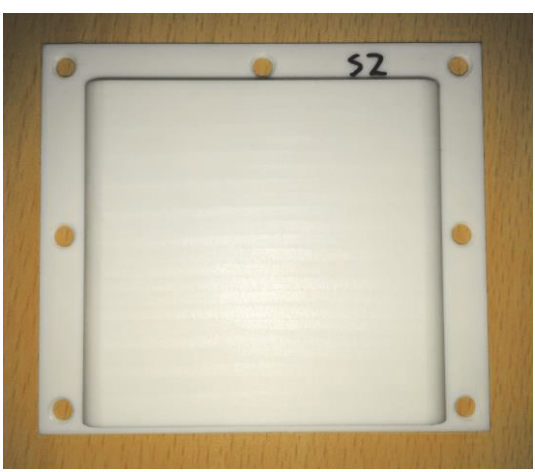

(a) Smooth-walled radome outer surface

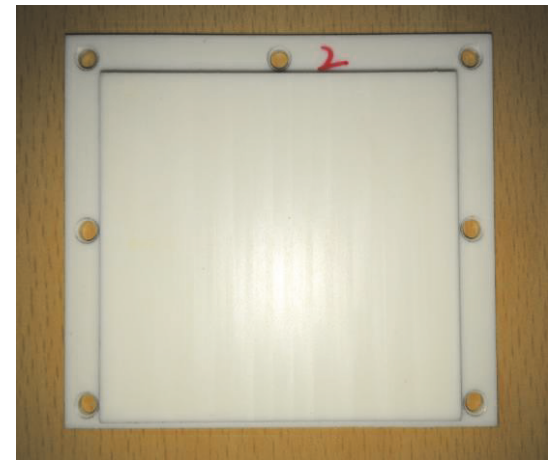

(c) Nondefected corrugated radome outer surface

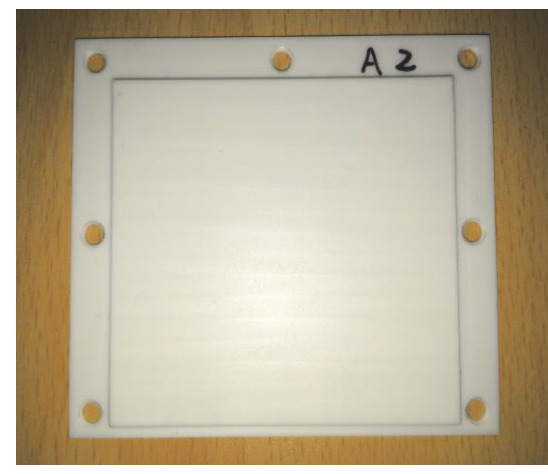

(e) Defected corrugated radome outer surface

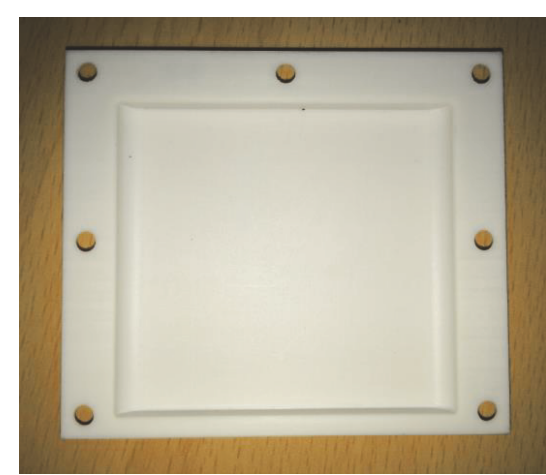

(b) Smooth-walled radome inner surface

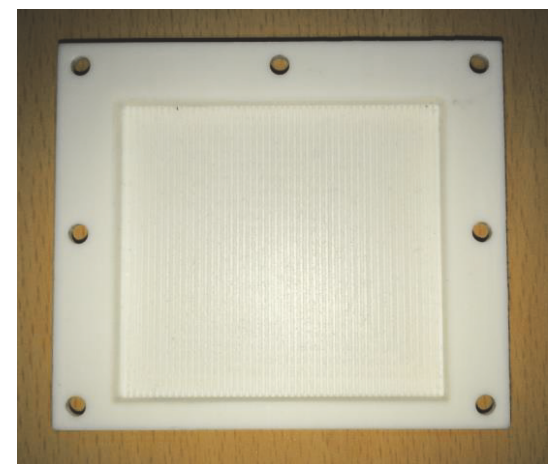

(d) Nondefected corrugated radome inner surface

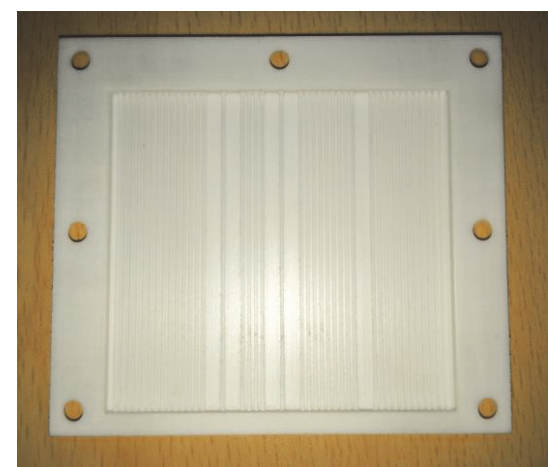

(f) Defected corrugated radome inner surface

Figure 17: Photographs of the fabricated radomes.

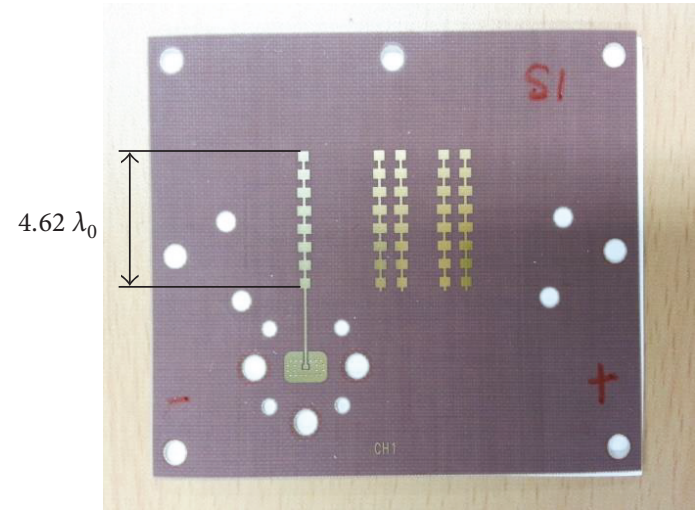

FIGURE 18: Photograph of a fabricated five-channel series-fed patch array.
TABLE 6: Radome thickness, height, and measured beam width.

\begin{tabular}{lccc}
\hline Model & $\begin{array}{c}d \\
(\mathrm{~mm})\end{array}$ & $\begin{array}{c}h \\
(\mathrm{~mm})\end{array}$ & $\begin{array}{c}\text { Beam width } \\
\text { (degrees) }\end{array}$ \\
\hline No radome (antenna only) & - & - & 129 \\
Smooth-walled radome & 1 & 4 & 99 \\
Nondefected corrugated radome & 2 & 4 & 128 \\
Defected corrugated radome & 1 & 2 & 152 \\
\hline
\end{tabular}

are numbered as channels one to five from the left. All of those five antennas achieve $3 \mathrm{dBi}$ beam widths of 150 degrees when covered with defected corrugated radome. Measurements were performed for all five channels; however, only 


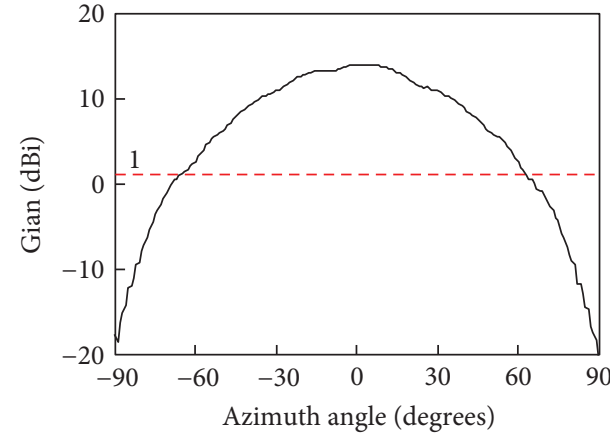

(a) No radome

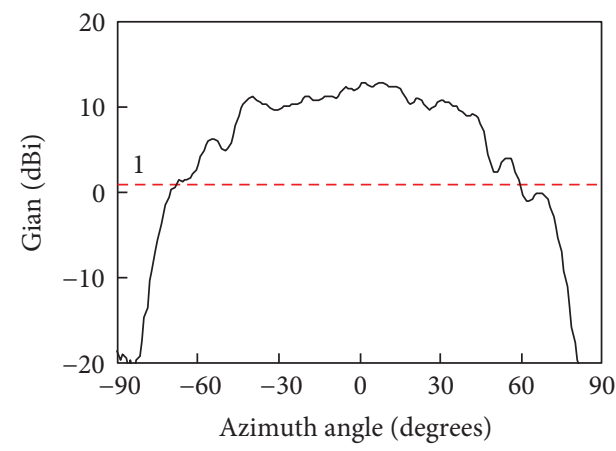

(c) Nondefected corrugated radome

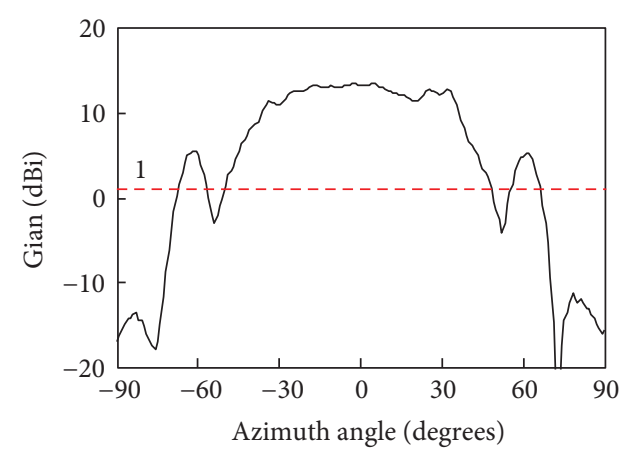

(b) Smooth-walled radome

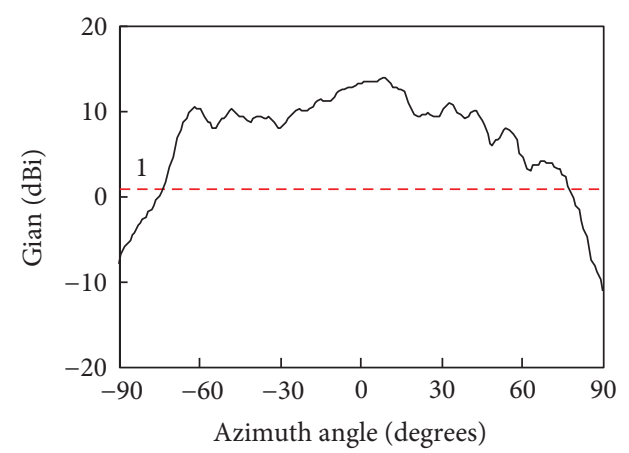

(d) Defected corrugated radome

FIGURE 19: Measured radiation patterns of Ch. 3 at $76.5 \mathrm{GHz}$ when radomes having different inner surfaces are used.

the data with the third channel are shown because they are least affected by the edges.

Figure 19 shows the measured radiation patterns of different radomes shown in Figure 17. In the preceding simulation results, the $3 \mathrm{dBi}$ gain is used as the criteria when deciding beam width, yet here, $1 \mathrm{dBi}$ gain is used. That is because a microstrip-to-waveguide transition introduces a $2 \mathrm{~dB}$ loss. Without a radome, the antenna has 129-degreewide beam width. With smooth and nondefected corrugated radomes whose $h$ is $4 \mathrm{~mm}$ and $d$ values are $1 \mathrm{~mm}$ and $2 \mathrm{~mm}$, respectively, beam width gets narrower. However, in the earlier section, nondefected corrugated radome with $d=1 \mathrm{~mm}$ and $h=2 \mathrm{~mm}$ shows wider beam width. Antenna covered with defected corrugated radome shows the widest beam width, and it is 152 degrees. This radiation pattern is dislocated from the axis of the symmetry. The reason is that the locations of the defected region are not uniform above the radiating antenna.

\section{Conclusion}

In this paper, design guidelines on defected corrugated radomes for BSD radars are given which increase beam widths. To achieve large beam widths, slowly varying magnitude and rapidly varying phase are needed. It is shown that by introducing defects on the corrugated surface, those two conditions are met, whereby 35\% beam width increase is realized compared with a simple smoothwalled radome.

\section{Conflicts of Interest}

The authors declare that there is no conflict of interests regarding the publication of this article.

\section{References}

[1] M. I. Skolnik, Introduction to RADAR Systems, McGraw-Hill, New York, NY, USA, 2nd edition, 1980.

[2] U.S. Department of Defense, MIL-R-7705B, General Specification for Radomes, U.S. Government Printing Office, 1975.

[3] R. Collin and J. Brown, "The design of quarter-wave matching layers for dielectric surfaces," Proceedings of the IEE Part C: Monographs, vol. 103, pp. 153-158, 1956.

[4] T. Morita and S. Cohn, "Microwave lens matching by simulated quarter-wave transformers," IRE Transactions on Antennas and Propagation, vol. 4, pp. 33-39, 1956.

[5] J. Mayhan and A. Simmons, "A low sidelobe Ka-band antenna-radome study," IEEE Transactions on Antennas and Propagation, vol. 23, pp. 569-572, 1975.

[6] A. Philippe, "Reflection and transmission of radio waves at a dielectric slab with variable permittivity," IEEE Transactions on Antennas and Propagation, vol. 21, pp. 234-236, 1973.

[7] R. U. Nair, S. Shashidhara, and R. Jha, "Novel inhomogeneous planar layer radome design for airborne applications," IEEE Antennas and Wireless Propagation Letters, vol. 11, pp. 854856, 2012.

[8] M. Khalaj-Amirhosseini, "Wideband flat radomes using inhomogeneous planar layers," International Journal of Antennas and Propagation, vol. 2008, Article ID 636047, 6 pages, 2008. 
[9] Y. Shibano and S. Yamashita, Radome with Matching Layers, U.S. Patent 3780 374, 1973.

[10] F. Fitzek and R. H. Rasshofer, "Automotive radome designreflection reduction of stratified media," IEEE Antennas and Wireless Propagation Letters, vol. 8, pp. 1076-1079, 2009.

[11] T. Tamir and S. Peng, "Analysis and design of grating couplers," Applied Physics, vol. 14, pp. 235-254, 1977.

[12] R. E. Collin, Field Theory of Guided Waves, Wiley, New York, NY, USA, 1990.

[13] J. D. Kraus and R. J. Marhefka, Antennas for All Applications, pp. 501-528, McGraw-Hill, 3rd edition, New York, NY, USA, 2002, Chapter 15. 


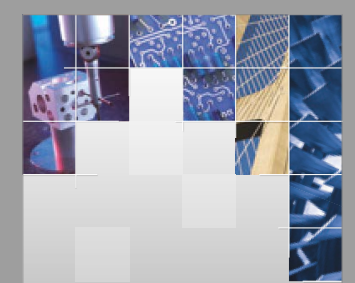

\section{Enfincering}
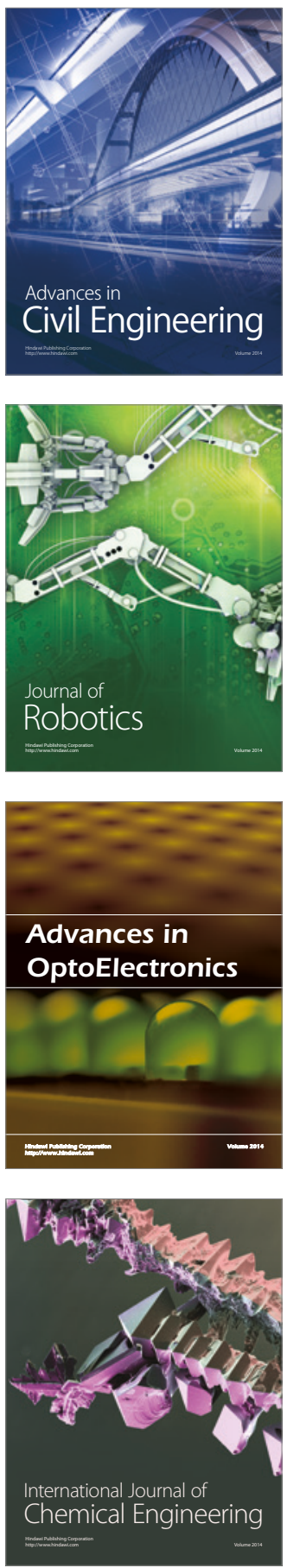

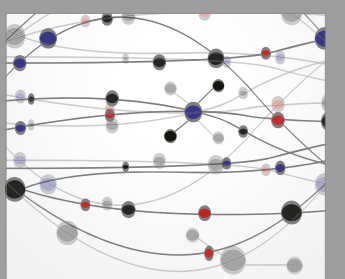

The Scientific World Journal

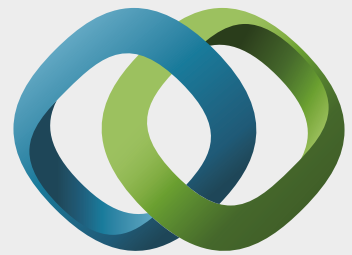

\section{Hindawi}

Submit your manuscripts at

https://www.hindawi.com
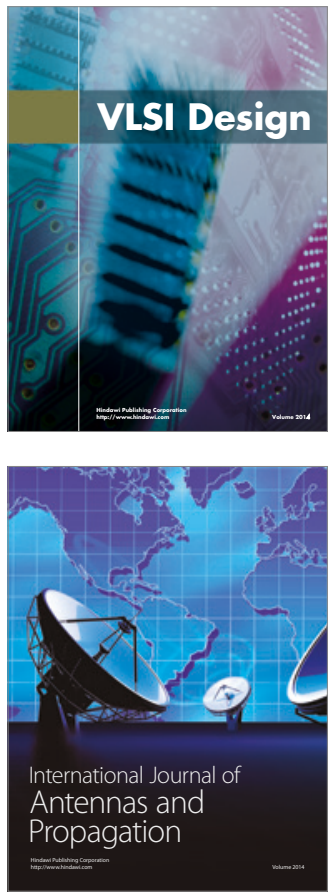

\section{Rotating}

Machinery
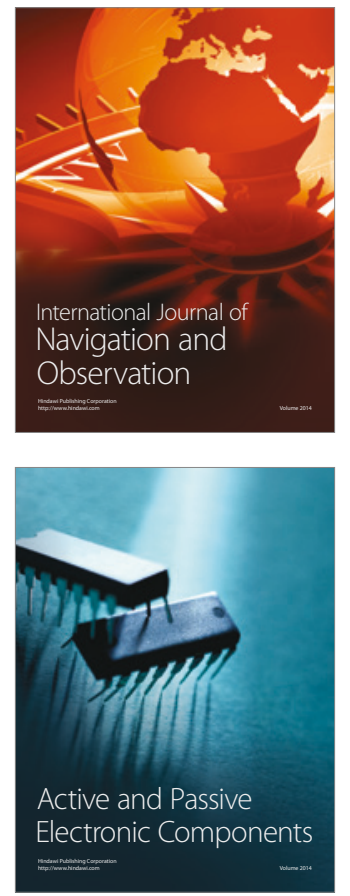
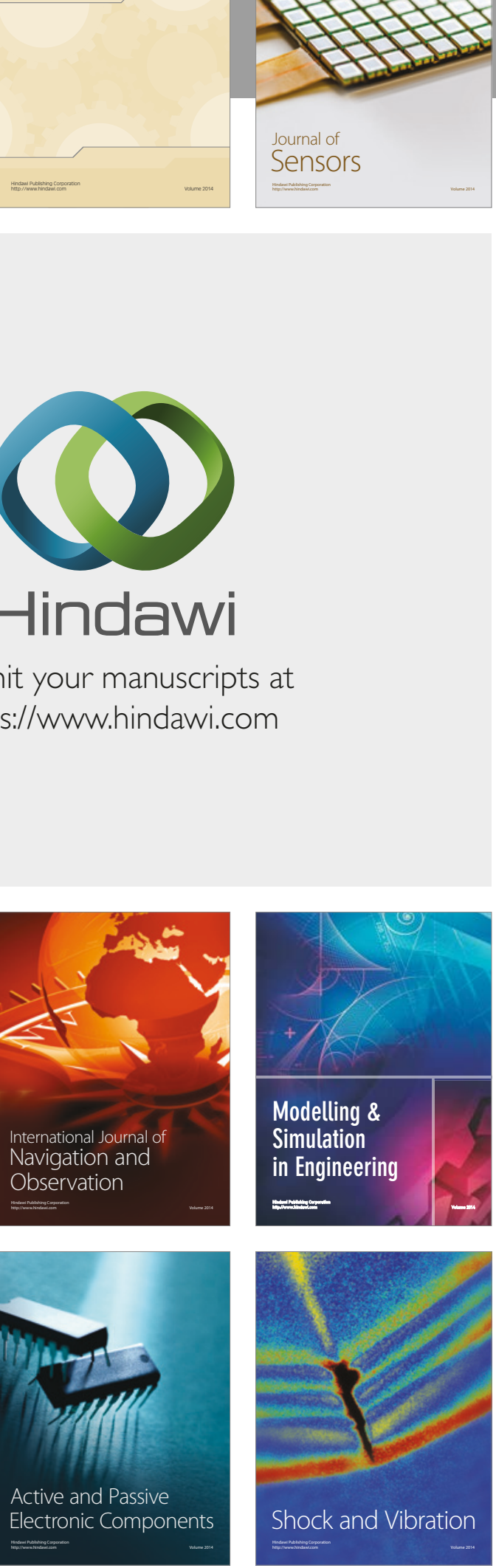
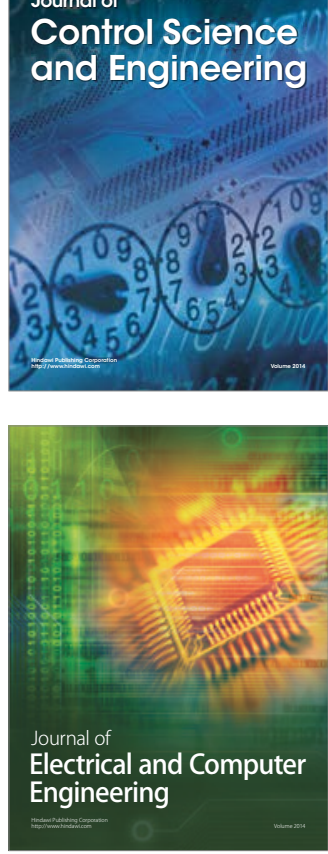

Distributed

Journal of

Control Science

and Engineering
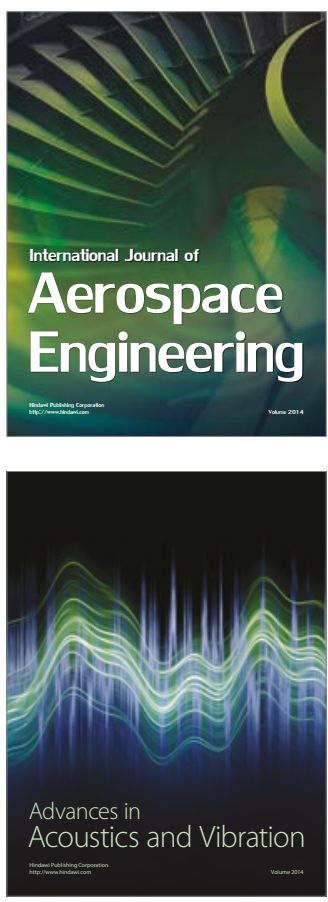

Sensor Networks 